\section{Variability of State-Approved Emergency Medical Services Drug Formularies}

\author{
*Theodore $R$. Delbridge, $M D$, Vincent $P$. Verdile, \\ MD, Thomas E. Platt, NREMT-P \\ University of Pittsburgh, Division of Emergency Medicine; \\ Albany Medical Center, Department of Emergency \\ Medicine; Center for Emergency Medicine of \\ Western Pennsylvania, Pennsylvania
}

Purpose: Recent national initiatives advocate development of standard practices for out-of-hospital emergency care. Regional practice variations place demands on educational programs and personnel. The objective of this study was to assess the variability of state-approved EMS drug formularies.

Methods: The design was a prospective, unstructured survey. State EMS directors or medical directors were requested, via letter, to forward a copy of their state's approved advanced life support (ALS) drug list. When necessary for clarification or to achieve a response, telephone contact was made.

Results: Responses $(\mathrm{n}=50$ ) were received from all 50 state EMS offices between June 1992 and December 1993. There are six categories of state-approved ALS drug lists: 1) no approved drug list, all formulary decisions made locally ( $n=22)$; 2) suggested medications via state protocols, all formulary decisions made locally $(\mathrm{n}=2)$; 3) mandatory formulary to be available to all ALS personnel, no local decisions $(\mathrm{n}=1)$; 4) approved formulary utilized at local medical director's discretion ( $\mathrm{n}=13$ ); 5) minimum required formulary, plus optional approved formulary utilized at local medical director's discretion $(n=5)$; and 6$)$ minimum required formulary, plus any additional drugs at local medical director's discretion $(n=7)$. Some states also have formulary addenda for interhospital transfers $(n=4)$, air medical services $(n=2)$, and pediatric teams $(n=1)$. A total of 79 drugs either are specifically required and/or approved in state EMS formularies. However, 42 drugs each are approved in fewer than six states. The most commonly required drugs ( 12 states each) are: atropine, epinephrine, $50 \%$ dextrose, lidocaine, and naloxone. Drugs approved by only one state $(n=20)$ include apresoline, butorphanol, phenylephrine, and tetanus antitoxin. No paralytic agents are included in any statewide formulary. State-approved EMS drug formularies list an average of $29 \pm 19.0$ medications. The Department of Transportation, National Standard Curriculum for paramedics includes 22 drugs.

Conclusions: State-approved, EMS drug formularies vary greatly. This variability has implications for developing standardized EMS practices and educational program curricula.

\section{A Prospective, Random Trial of Epinephrine, Metaproternol, and Epinephrine and Metaproterenol in the Prehospital Treatment of Adult Asthma}

\author{
Mark Quadrel, MD, *Robert F Lavery, BA, MICP, \\ Michael Jaker, MD, Bartholomew J. Tortella, \\ MTS, MD, FACS, Ronald P. Cody, EdD \\ New Jersey Trauma Center, University EMS, University \\ Emergency Department, UMDNJ, New Jersey Medical \\ School, Newark, New Jersey
}

Purpose: The purpose of this study was to compare the efficacy and adverse response of three regimens for the prehospital treatment of adult asthma.

Methods: Adult asthmatics (18-50 yrs) in the field were assigned randomly into one of three groups: 1) metaproterenol nebulization (MET);2) subcutaneous epinephrine (EP1); or 3) epinephrine and metaproterenol together (BOTH). Pre/post treatment PEAK flow measurements and vital signs were measured. Primary outcome variables were final PEAK $\left(\right.$ PEAK $\left._{\mathrm{F}}\right)$ and change in PEAK ( $\left.\operatorname{PEAK}_{\Delta}\right)$. Adverse response was defined as $\geq 15 \%$ increase in heart rate and/or blood pressure from baseline. Treatment failure was defined as no change or a decrease in $\mathrm{PEAK}_{\mathrm{F}}$.

Results: A total of 187 patients were enrolled with 154 (78\%) evaluable. There were no group differences for all of the demographic or pretreatment physiologic measures.

\begin{tabular}{lcccccccc} 
& \multicolumn{9}{c}{ AR } & & \multicolumn{3}{c}{$R_{x}$} \\
& $n$ & $\begin{array}{c}\text { PEAK } \\
(\times x / \times x x)\end{array}$ & $\pi(\%)$ & HR & SBP & OBP & $F$ \\
MET & 49 & 71.4 & $1(2)$ & 0 & 1 & 1 & $1(2)$ \\
EPI & 52 & 71.4 & $11(22)$ & 8 & 5 & 3 & $5(9.6)$ \\
BOTH & 53 & 79.0 & $12(23)$ & 5 & 5 & 2 & $8(15)$
\end{tabular}

( $n=$ evaluable records; $A R=$ adverse response; SBP = systolic blood pressure; $\mathrm{R}_{\alpha}=$ treatment; $\mathrm{DBP}=$ diastolic blood pressure; $F=$ failure; $n[\%]$ )

PEAK $_{\triangle}$ and PEAK $_{F}$ were not significantly different by one-way analysis of variance (ANOVA PEAK $: F=0.35$, $\left.p=0.71 ; \operatorname{PEAK}_{\triangle}: \mathrm{F}=0.20, p=082\right)$, or by analysis of covariance (ANCOVA - both parameters $\mathrm{F}=0.05$, $p=0.95)$. The power to detect a $25 \%$ difference in $\mathrm{PEAK}_{\mp}$ was $85 \%$.

Conclusions: Metaproterenol alone is as effective as subcutaneous ( $S Q$ epinephrine alone or in combination with SQ epinephrine in the treatment of moderate to severe exacerbation of adult asthma in the first 10-15 minutes. Because of the low adverse response profile and small number of treatment failures, metaproterenol should be the first-line treatment in the prehospital care of adult asthma. 